\title{
PRIORITIZING THE FACTORS AFFECTING THE SELECTION OF MACHINES USED IN DIGITAL TWIN PROCESSES: A BUSINESS EXAMPLE
}

\author{
DOI: 10.17261/Pressacademia.2021.1442 \\ PAP- V.13-2021(31)-p.116
}

\section{Ezgi Demir}

Piri Reis University, Faculty of Economics and Administrative Sciences, Department of Management Information Systems, Istanbul, Turkey. dem_ezgi@hotmail.com, ORCID: 0000-0002-7335-5094

To cite this document

Demir, E. (2021). Prioritizing the factors affecting the selection of machines used in digital twin processes: a business example. PressAcademia Procedia (PAP), V.13, 116.

Permanent link to this document: http://doi.org/10.17261/Pressacademia.2021.1442

Copyright: Published by PressAcademia and limited licensed re-use rights only.

\section{ABSTRACT}

Purpose- Recently, digital twin technologies have been frequently used in projects in the digital transformation processes of businesses. In digital twin processes; there are many different reasons for choosing the machines to be used, such as cost, purpose of use of the business, technology level of the business. In this study, it is aimed to prioritize the factors affecting the machines to be used in digital twin processes. Methodology- Digital twin processes has brought the uncertainty process for businesses. This situation requires decision making under uncertainty. The decision-making process under uncertainty requires the examination of many value judgments together.Digital twin processes has brought the uncertainty process for businesses. This situation requires decision making under uncertainty. The decision-making process under uncertainty requires the examination of many value judgments together. For this reason, in order to make the right decisions in projects, the prioritization of the factors affecting the digital transformation processes was carried out by taking expert opinion. In this study, the Spherical Fuzzy AHP method, which was newly introduced to the literature, has been used. For this purpose, 5 important criteria were determined from the literature and expert opinions. Three expert opinions have been received.

Findings- The results of the model have showed that simulation process is the most important criteria for the selection of machines used in digital twin processes. And sensor based systems are the least important criteria for digital twin processes.

Conclusion- In this study, prioritizing factors in the selection of machines used in digital twin processes have been examined. For this purpose the mechanization processes to be used in digital twin processes bring many control points in the background. In this study, the prioritization of the machinery and equipment to be used has been made by taking expert opinion. Opinions have been received from 3 experts in the field. The Spherical Fuzzy AHP method, which is one of the fuzzy decision-making sets, which has been recently introduced to the literature, has been used. In the study, it is expected to contribute to the literature by using digital twin technology for the first time with a multi-criteria decision-making methodology.

Keywords: Digital twin, MCDM, technology, process, Spherical Fuzzy AHP JEL Codes: 031, M15, C44

\section{REFERENCES}

Feng Xiang, Yuan yuan Huang, Zhi Zhang, Ying Zuo, (2020). Digital twin driven energy-aware green design,vEditor(s): Fei Tao, Ang Liu, Tianliang Hu, A.Y.C. Nee,Digital Twin Driven Smart Design,Academic Press, 165-184, ISBN 9780128189184.

Li, Lianhui, Mao, Chunlei, Sun, Hongxia, Yuan, Yiping and Lei, Bingbing, (2020). Digital Twin Driven Green Performance Evaluation Methodology of Intelligent Manufacturing: Hybrid Model Based on Fuzzy Rough-Sets AHP, Multistage Weight Synthesis, and PROMETHEE II", Complexity in Economics and Business, Vol. 2020, Article ID 3853925, 1-24.

Shanghua Mi, Yixiong Feng, Hao Zheng, Yong Wang, Yicong Gao, Jianrong Tan, (2021). Prediction maintenance integrated decision-making approach supported by digital twin-driven cooperative awareness and interconnection framework, Journal of Manufacturing Systems, V.58, 329-345, ISSN 0278-6125.

Sihan Huang, Guoxin Wang, Yan Yan, Xiongbing Fang, (2020). Blockchain-based data management for digital twin of product. Journal of Manufacturing Systems, V. 54, 361-371.

Wenjun Xu, Jia Cui, Lan Li, Bitao Yao, Sisi Tian, Zude Zhou, (2021). Digital twin-based industrial cloud robotics: Framework, control approach and implementation. Journal of Manufacturing Systems, V. 58, 196-209. 